\title{
Image texture analysis of apples broken down in a mastication simulator prototype
}

\author{
Billy, L. ${ }^{1,2}$, Arvisenet, G. ${ }^{1}$, Poinot, P. ${ }^{1}$, Chevallier, S. ${ }^{2}$, Royer, G. ${ }^{4}$, Vigneau, E. ${ }^{3}$ and Prost, C. ${ }^{1}$ \\ ${ }^{1}$ ENITIAA - Food Aroma Research Group, Rue de la Géraudière, BP 82225, 44322 Nantes cedex 03, France \\ ${ }^{2}$ ENITIAA - GEPEA-UMR CNRS 6144, Rue de la Géraudière, BP 82225, 44322 Nantes cedex 03, France \\ ${ }^{3}$ ENITIAA - INRA - UMR Sensometrics Chemometrics, Rue de la Géraudière, 44322 Nantes cedex 03, France \\ ${ }^{4}$ ESA - Laboratoire Grappe, 55 Rue Rabelais, BP 30748, 49007 Angers cedex 01, France \\ Corresponding author: arvisenet@enitiaa-nantes.fr
}

An artificial mouth has been developed, in the food aroma research group of ENITIAA, to study aroma release from hard products. This study was undertaken to validate this apparatus with apple as an example of a hard product. Before analyzing apple volatile compounds by this new method, it was necessary to study the importance of food breakdown and to compare it with that obtained in the human mouth. With this aim, an image analysis method was developed. Different crushing parameters were applied to Golden Delicious apples in an experimental procedure designed to obtain different breakdown states. Images of apples broken down in the artificial mouth were compared with images of apples chewed in the human mouth. Two conditions were found to lead to apple states very close to that obtained after chewing in the human mouth. The repeatability of the crushing in these conditions was shown to be satisfactory.

Keywords: apple, artificial mouth, image texture analysis, oral breakdown

\section{Introduction}

Fruit quality strongly depends on organoleptic properties. Among these, the visual aspect influences the consumer's food choice but flavor and texture induce consumers to buy a fruit variety again. To study food aroma, two approaches have been developed: sensory and instrumental analyses. Although sensory analysis is the reference method, it is not always easy to apply because it is long and expensive, and depends very much on the panel. Extraction methods are easier to use and more reproducible but most of them do not take into account the changes occurring during the eating process. The release of volatile compounds can be markedly affected by these changes, particularly in hard foods such as apples. This is probably why, in the literature, dynamic headspace analysis has been largely used to study the aroma compounds of food because it partially reproduces the way volatile compounds are released from food in the mouth. Nevertheless, it is not always the most suitable technique. For example, Mehinagic et al. (2004) studied the influence of dynamic headspace conditions on aroma release from apple slices. The extracted quantity of total odorant volatiles increased with the temperature and purge time. The conditions leading to the highest extracted quantity of total odorant volatiles also induced a distortion of the aroma, with the development of a cooked apple aroma.

Therefore, new instrumental methods are being developed to extract volatile compounds in conditions as similar as possible to the mouth. They are described as "artificial mouths" or "mastication simulators". They reproduce the changes occurring during the consumption of foods. These changes induce the release of volatile compounds, which are immediately trapped then analyzed. A few model mouth systems have been developed by several authors to study liquids (van Ruth et al., 2000 and 2004 ; Rabe et al., 2004) or viscous model products (Odake et al., 2000), and also "semi-solid" natural foodstuffs like vegetables (van Ruth et al., 1994, 1995, 2000). In these studies, the products were either stirred or crushed with a plunger making up and down screwing movements. The authors did not describe the final state of the semi-solid food after mastication by the plunger. 
To design our artificial mouth, our approach was to take into account the breakdown of food as well as the release of aroma compounds. Since it is widely known that flavor release depends on food structure and texture (Carr et al., 1996 ; Karlsen et al., 1999), we considered that efficient crushing may lead to a the most important flavor release. To determine what efficient crushing is, the best reference seems to be the crushing obtained by mastication in the human mouth. Thus, we decided to search for crushing conditions that would produce apple samples in the same breakdown state as after being crushed in the human mouth.

This study was undertaken to determine the experimental conditions that would put apples into such a breakdown state. We developed an image analysis method to compare apples normally chewed by people and apples crushed in the artificial mouth. This method allowed us to take into account the variability in human mastication.

\section{Materials and Methods \\ Apples}

Golden Delicious apples, harvested in 2005, were purchased at a local supermarket one week before analysis. Apples were stored at $2{ }^{\circ} \mathrm{C}$ until the day before measurements were made and were brought up to room temperature $24 \mathrm{~h}$ before analysis.

\section{Apples chewed in the human mouth}

Three males and three females, aged between 20 and 60 years, were chosen in order to take into account any possible changes in dentition with age. For each apple, subjects were asked to peel it, crunch a piece of it, chew it normally and then spit out the bolus at the point when swallowing would normally have been triggered. To take into account apple texture variability, four randomly selected apples were used and two apple pieces were crunched in each of them. In total, each subject chewed eight apple pieces, which were mixed before being photographed. Two images were acquired for a same mixture. Two sessions were carried out which led twenty-four pictures for apple breakdown in the human mouth.

\section{Apples chewed in the artificial mouth}

For each experiment, the texture uniformity of four randomly selected fruits was checked by penetrometry. Then, these apples were peeled, cut in half and their cores were discarded. Equal longitudinal slices $(0.8 \mathrm{~mm}$ thick and $2 \mathrm{~cm}$ long) were cut from each apple. 68 grams of apple and $15 \mathrm{~mL}$ of water, purified by a milli-Q system (Millipore Corp., Molsheim, France), were introduced into the container of the artificial mouth. This device consists of a hermetically-sealed container in which products were broken down by a compression movement and a rotation movement. The device was maintained at $37^{\circ} \mathrm{C}$ by an outer layer. Volatile compounds can be trapped simultaneously with this breakdown (a function not used in this study). Assays were carried out according to an experimental procedure chosen to give different breakdown states of apple slices. The parameters were: compression movement frequency $(25,50,75$ chews per minute), two rotation movement speeds (with a factor of ten between them; they will be called "1" and "10" in the present document) and time (10, 20, 30 minutes). In each condition, two replicates were carried out and two pictures were acquired per replicate. In total, seventy-two pictures were analyzed for apple destruction in the artificial mouth.

\section{Data acquisition}

The imaging system was composed of a CDD camera (SONY DFW-SX910, NOESIS, France) equipped with a lens (focal length of $9 \mathrm{~mm}$ ) and mounted on a photographic bench (Figure 1). The camera was connected to a PC with a bus IEEE-1394. The lighting system was composed of two white light tubes inside two semi-spherical black chambers, thus providing a homogeneous direct visible light. Each sample of apple was spread out in a Petri dish to present a flat surface and a regular thickness. 
Digitized images of $512 \times 512$ pixels corresponding to a sample surface of $5 \times 5 \mathrm{~cm}$ were chosen. We considered this surface to be representative of the whole apple sample. Two images were acquired per sample.

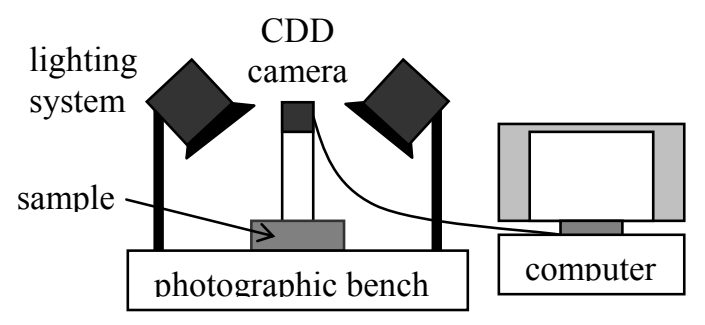

Figure 1: Image acquisition system

\section{Image texture analysis}

Images, initially acquired in an RGB system, were transformed into grayscale images. Hence, an image was stored in a matrix containing brightness values associated to each pixel, ranging from 0 (black) to 255 (white). An example is shown in Figure 2.

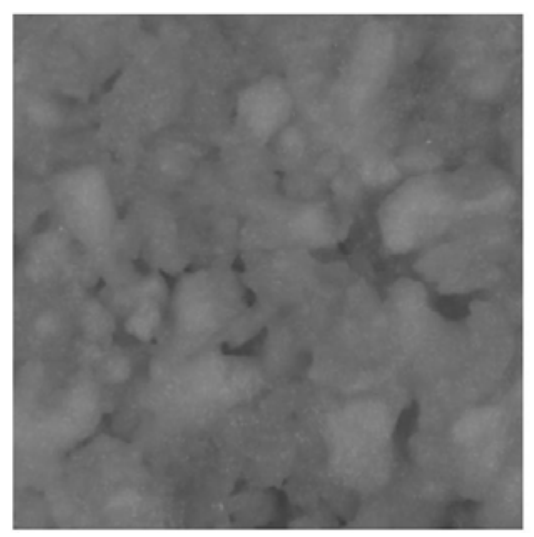

Figure 2: Grayscale image of chewed Golden apple obtained for a given assessor

The images of the chewed apples can be characterized by their smoothness, or their coarseness, which depends on the number and the size of the apple pieces more or less distinguishable from a puree. From a mathematical point of view, we aimed to characterize the texture of the images, this being described by some authors as an attribute representing the spatial arrangement of the gray levels of the pixels in a region of the image (Tuceryan and Jain, 1998; Bharati et al., 2004). The methodology used, which is probably the most frequently cited method for statistical texture analysis, was based on the extraction of various textural features from a gray level co-occurrence matrix (GLCM).

A GLCM is a matrix of size $\mathrm{Ng} \times \mathrm{Ng}$ ( $\mathrm{Ng}$ is the number of gray levels) defined for a displacement of a distance $\delta$ along a given direction $\theta$. The entry $\mathrm{P}_{\delta, \theta}(i, j)$ of this matrix is the number of occurrences of a pair of gray levels, $i$ and $j$, for the specified displacement. Figure 3 gives an illustration of the construction of the GLCM. 


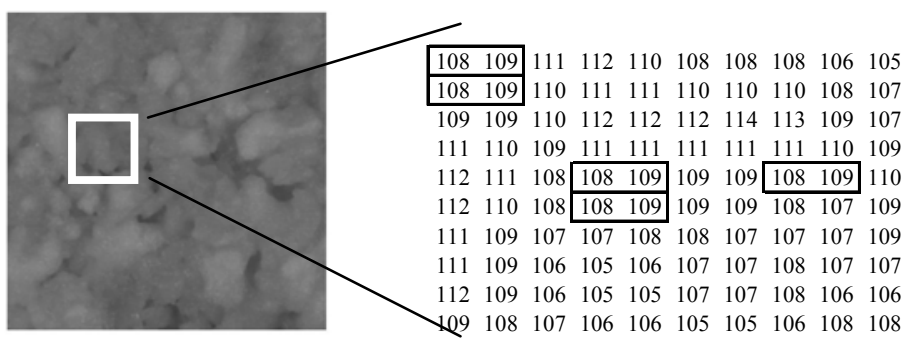

initial image portion of the intensity matrix

$\begin{array}{cccccccccccc} & 105 & 106 & 107 & 108 & 109 & 110 & 111 & 112 & 113 & 114 \\ 105 & 2 & 2 & 1 & 0 & 0 & 0 & 0 & 0 & 0 & 0 \\ 106 & 4 & 2 & 1 & 1 & 0 & 0 & 0 & 0 & 0 & 0 \\ 107 & 0 & 1 & 6 & 3 & 2 & 0 & 0 & 0 & 0 & 0 \\ 108 & 0 & 2 & 5 & 6 & 5 & 0 & 0 & 0 & 0 & 0 \\ 109 & 0 & 2 & 2 & 3 & 5 & 3 & 2 & 0 & 0 & 0 \\ 110 & 0 & 0 & 0 & 3 & 2 & 2 & 1 & 1 & 0 & 0 \\ 111 & 0 & 0 & 0 & 1 & 2 & 3 & 5 & 1 & 0 & 0 \\ 112 & 0 & 0 & 0 & 0 & 1 & 2 & 1 & 2 & 0 & 1 \\ 113 & 0 & 0 & 0 & 0 & 1 & 0 & 0 & 0 & 0 & 0 \\ 114 & 0 & 0 & 0 & 0 & 0 & 0 & 0 & 0 & 1 & 0\end{array}$

GLCM obtained for this portion $\left(\delta=1, \theta=0^{\circ}\right)$.

Figure 3: From the image to the GLCM

Once the GLCM is defined, various textural descriptors can be evaluated. We considered the most widely used ones i.e. the energy, the entropy, the contrast, the inverse differential moment as well as the correlation, which are defined as follows:

$$
\begin{aligned}
& \text { energy }=\sum_{i=1}^{N_{g}} \sum_{j=1}^{N_{g}} P_{\delta, \theta}(i, j)^{2} \\
& \text { entropy }=\sum_{i=1}^{N_{g}} \sum_{j=1}^{N_{g}} P_{\delta, \theta}(i, j) \log \left(P_{\delta, \theta}(i, j)\right) \\
& \text { contrast }=\sum^{N_{g}-1} k^{2}\left[\sum_{i=1}^{n_{g}} \sum_{j=1}^{n_{g}} P_{\delta, \theta}(i, j)\right] \\
& \text { inv.diff.moment }=\sum_{i=1}^{N_{g}} \sum_{j=1}^{N_{g}} \frac{1}{1+(i-j)^{2}} P_{\delta, \theta}(i, j) \\
& |\mathrm{i}-\mathrm{j}|=\mathrm{k}
\end{aligned}
$$

correlation $=\sum_{i=1}^{N_{g}} \sum_{j=1}^{N_{g}} \frac{\left(i-\mu_{x}\right)\left(j-\mu_{y}\right) P_{\delta, \theta}(i, j)}{\sigma_{x} \sigma_{y}}$ where $\mu_{x}\left(\right.$ resp. $\left.\mu_{y}\right)$ and $\sigma_{x}\left(\right.$ resp. $\left.\sigma_{y}\right)$ are the mean and the standard deviation of the GLCM marginal distributions by row (resp. by column).

From the definitions given above, we can expect that the energy and the entropy are two linked parameters, like the contrast and the inverse differential moment with an opposite behavior. As this was corroborated by the data, further data analyses were based only on the energy, contrast and correlation parameters.

Moreover, these textural parameters were evaluated for different displacements. As the chewed apple images seemed to be isotropic, we always considered a horizontal displacement direction $\left(\theta=0^{\circ}\right)$ but with several distances, varying from one pixel $(\delta=1$, about $0.1 \mathrm{~mm}$ on the images) to 150 pixels. Due to the high redundancy of the information obtained between some distances, we finally selected $\delta=1,10$ and 30 in order to be able to evaluate the texture of the images at different levels of sharpness.

Another aspect deals with the pretreatment of the images. Unlike the images obtained from the human mouth, the images obtained with the artificial mouth needed to be pretreated. In fact, the addition of water to the apparatus, to mimic saliva, produced reflected light having a great influence on the textural parameters, such as the contrast. The amount of added water had been determined to correspond to the volume of saliva used by people to chew apple, but subjects swallowed the saliva before spitting out chewed apple whereas, in the artificial mouth, we could not remove the water. 
The pretreatment of this collection of images consists, in summary, of detecting the edges associated with the reflections (using a special procedure included in the Image toolbox of Matlab 7.0®The Mathworks) then, identifying, from each pixel linked to an edge, a zone whose gray levels are higher than that of the reference pixel and finally applying a median filter on the region surrounding the delineated zone. Figure 4 shows the effect of this pretreatment on a specific image. The mathematical correction seemed satisfactory and was applied to all the images of the collection.
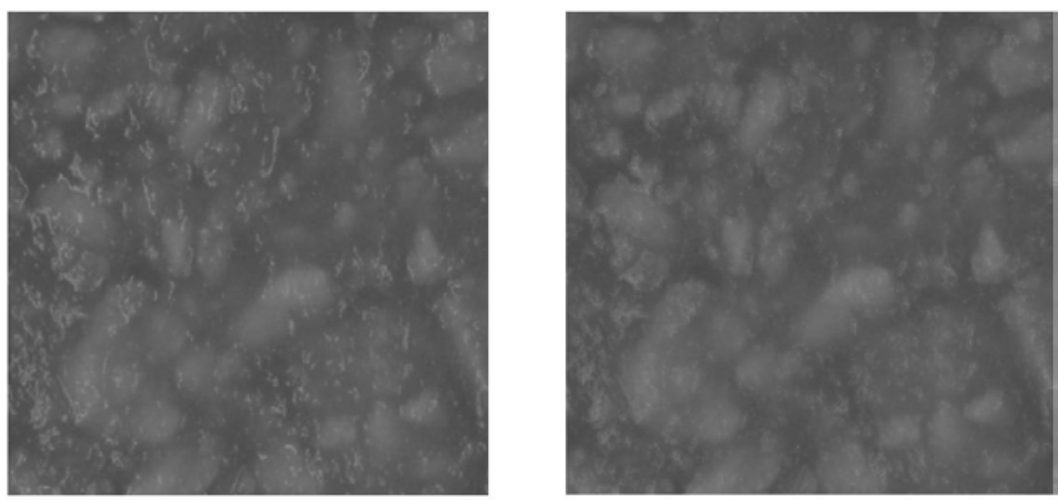

Figure 4: An image obtained with the artificial mouth before (left) and after (right) pretreatment

\section{Results and Discussion}

Collected data consist of nine parameters of texture extracted from each image. For the data treatment, firstly, we described the 24 images of apples chewed by subjects according to the selected texture parameters by means of a Principal Components Analysis. Thereafter, we projected the 72 images obtained with the artificial mouth on the first factorial plan and we evaluated the distances between the reference images (human mouth) and the supplementary ones (artificial mouth). The artificial mouth images close to the reference images enable the optimal crushing conditions for the apparatus to be identified.

The correlation circle and the configuration of the human mouth images, according to the two first principal components, are shown in Figure 5.
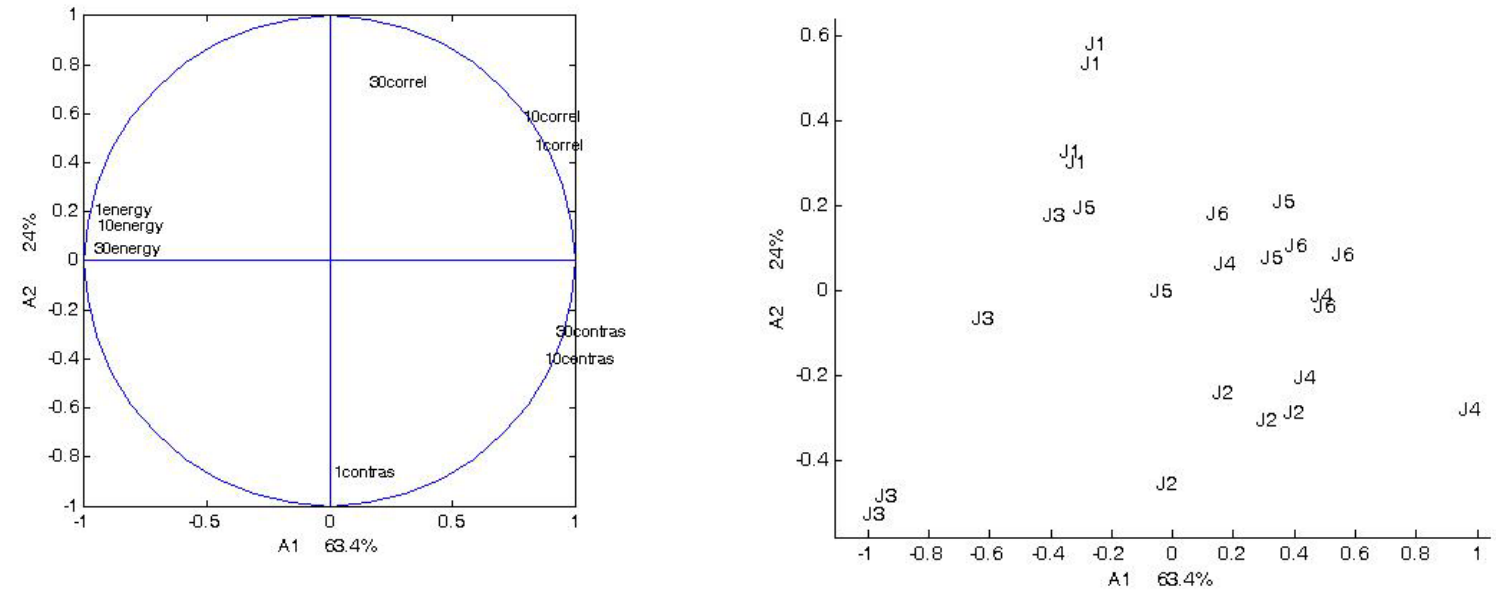

Figure 5: Principal Components Analysis of the images acquired with the human mouth, according to the nine parameters of texture: correlation circle (left) and configuration of the samples (right)

We can observe that the energy parameters (whatever the distance used for displacement) are redundant and opposite to the contrast and correlation parameters. 
The second principal component is especially linked to images with a high local contrast and low GLCM correlations. On this reference factorial plan, the images of the six subjects are quite well separated. The judge 3 (J3) produced highly chewed samples, like a puree, while judge 4 (J4) coarsely crunched the apples as illustrated in Figure 6. The repeatability of the image acquisition (same subject, same session) and of the subjects (across sessions) seems quite good (Figure 5).

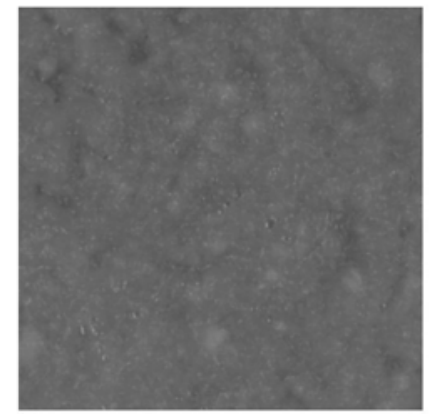

subject 3

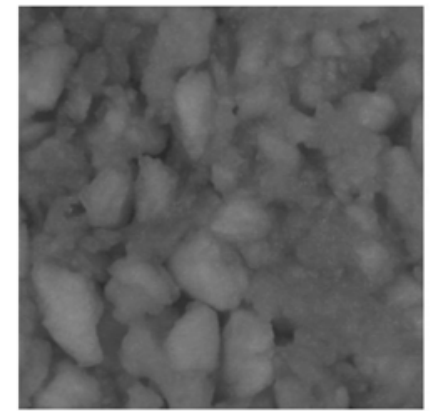

subject 4

Figure 6: Examples of variability in chewing efficiency for two different subjects

The projection of the images obtained with the artificial mouth onto the reference plan described above is illustrated in Figure 7. Each supplementary image is coded by the experimental conditions used (rotation movement speed - compression movement frequency time). It appears that most of the supplementary images have texture characteristics closer to the texture of images produced by subjects 2, 4, 5 and 6 than to those of subjects 1 and 3 . Nevertheless, in order to take into account as large a variability as possible between the subjects, all the images of the human mouth were involved in the evaluation of the distances between reference images and each of the supplementary images.

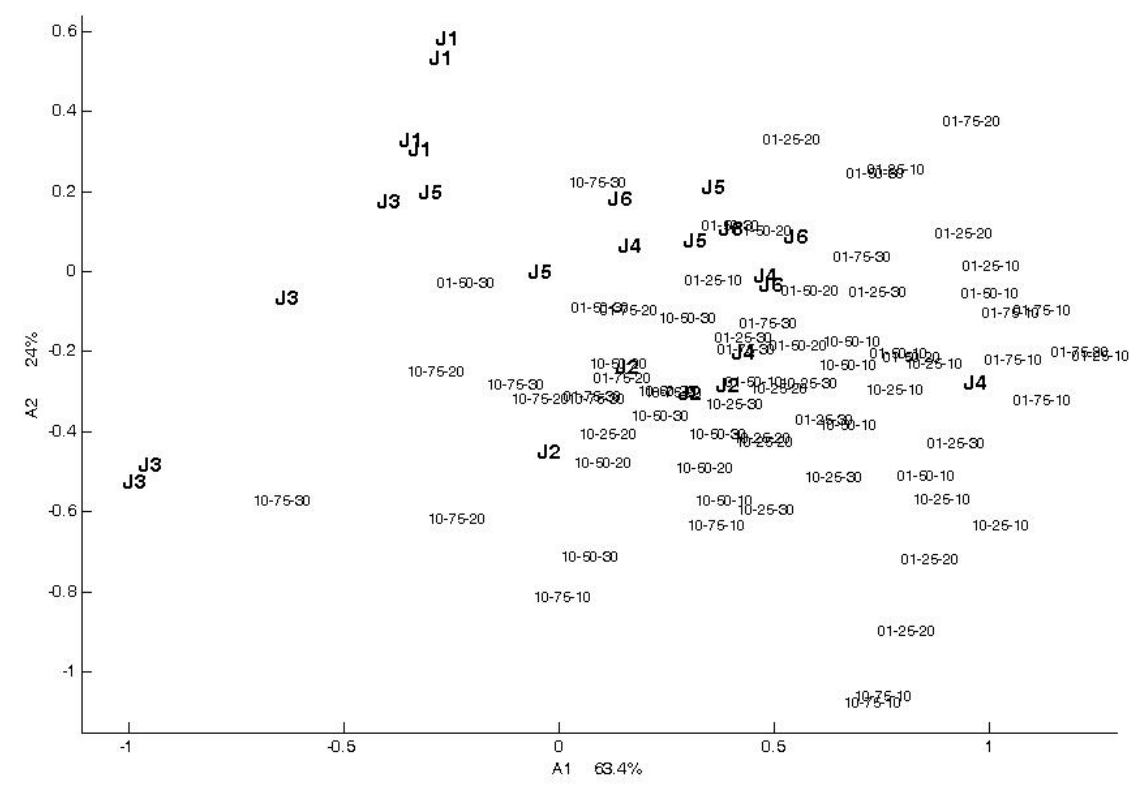

Figure 7: First factorial plan with the reference images acquired with the human mouth (J1 to J6) and the supplementary images acquired with artificial mouth conditions 
The distances were assessed according to the coordinates on the first two principal components. Firstly, the distances to the centroid of the four images associated with a specific subject were evaluated. After that, they were agglomerated for all the subjects. Figure 8 shows the resulting distances according to the experimental conditions of the artificial mouth. As the stability between the four repetitions performed for a given experimental condition is as important a criterion for us as the distances themselves, the rotation movement speed "1" appeared not to be very interesting, in general.

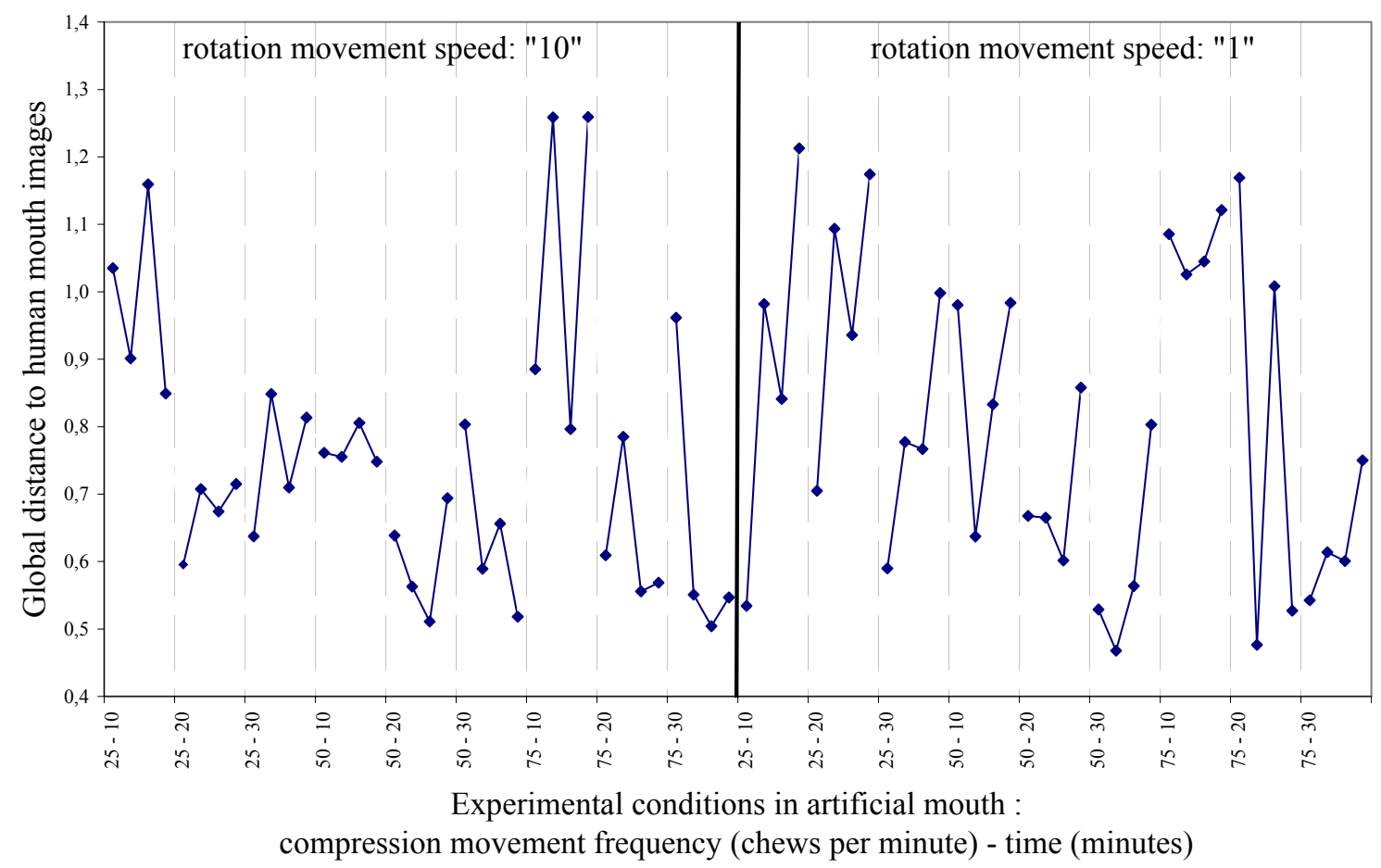

Figure 8: Global distances between the images of the artificial mouth, identified by their experimental conditions, and the human mouth images

The conditions retained corresponded more to a rotation movement " 10 " with a compression movement frequency of 25 or 50 chews per minute. A duration of 20 minutes seems to give the best results. Indeed, images at 10 minutes showed apples insufficiently broken down while 30 minutes of crushing led more a puree. Thus, the images closest to apple chewed in the human mouth were obtained at 20 minutes (Figure 9).

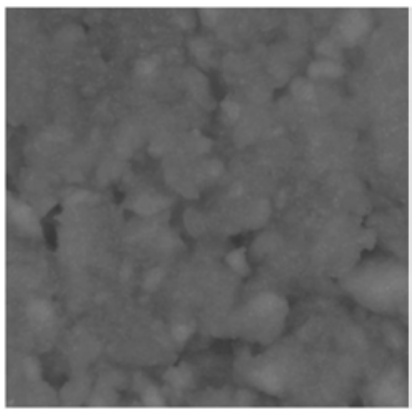

(a)

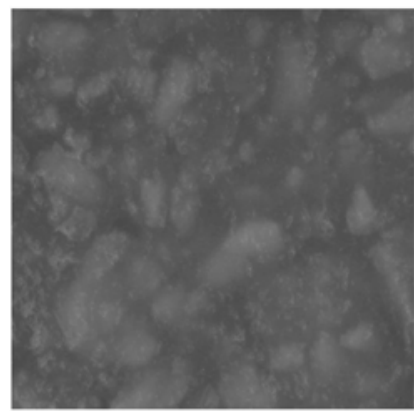

(b)

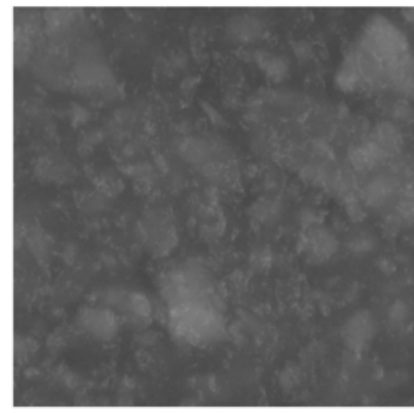

(c)

Figure 9: Comparison of images of apples crushed by subject 5 (a) and in the artificial mouth for 20 minutes, at rotation movement speed " 10 ", and at 25 (b) or 50 (c) chews per minute 


\section{Conclusion}

This study provided encouraging results indicating that the co-occurrence parameters give some information regarding apple fragment size after breakdown. This statistical treatment enabled us to validate our artificial mouth by showing that apple breakdown in it could be very close to the chewing in the human mouth. Two retained conditions led to an optimal crushing of apples in the artificial mouth, proved by images close to the reference images (human mouth). Moreover, the repeatability is satisfactory with these two conditions. However, it could be argued that the time (20 minutes) to achieve this breakdown is too long compared to what happens in the human mouth. In order to release apple volatile compounds in conditions as close as possible to the human mouth, it would be necessary to obtain such a breakdown in a shorter time. For that, it would be interesting to increase the rotation movement. Indeed, these first results show that the faster the rotation movement, the better the apples are broken down. Once better artificial mouth parameters are been determined, the apple volatile compounds will be studied.

\section{Acknowledgements}

We thank all members who devoted their precious time to the tests in human mouth. We are grateful to the students of ENITIAA, particularly Richard Batté, Emmanuelle Chartier and Guillaume Dahéron, for their statistical contribution. The authors give special thanks to Dominique Mitard, Olivier Rioux and Michelle Moreau for their valuable technical help. This research was supported by the Conseil Regional of Pays de Loire.

\section{References}

Bharati M.H., Liu J.J., MacGregor J.F., 2004. Chemometr. Intell. Lab. Syst. $72,57$.

Carr J., Baloga D., Guinard J.-X., Lawter L., Marty C., Squire C., 1996. The effect of Gelling Agent Type and Concentration on Flavour Release in Models Systems. In: Flavour-Food Interactions, Eds. McGorrin, R.J., Leland, J.V., American Chemical Society, 9: 99-108.

Karlsen A.M., Aaby K., Sivertsen H., Baardseth P., Ellekjaer M.R., 1999. Food Qual. Prefer. 10, 305. Mehinagic E., Prost C., Demaimay M., 2004. J. Agr. Food Chem. 52, 5175.

Odake S., Roozen J.P., Burger J.J., 2000. Biosci. Biotechnol. Biochem. 64, 2523.

Rabe S., Krings U., Berger. R.G., 2004. Chem. Senses. 29, 153.

Tuceryan M., Jain A.K., 1998. Texture Analysis. In: The Handbook of Pattern Recognition and Computer Vision ( $2^{\text {nd }}$ Edition), Eds. Chen C.H., Pau L F., Wang P S.P., World Scientific Publishing Co., 207-248.

van Ruth S.M., Roozen J.P., Cozijnsen J.L., 1994. Comparison of dynamic headspace mouth model systems for flavour release from rehydrated bell pepper cuttings. In: Trends in Flavour Research, Eds. Maarse H., van der Heij D.G., Elsevier Science, 59-64.

van Ruth S.M., Roozen J.P., Cozijnsen J.L., 1995. Food Chem. 53, 15.

van Ruth S.M., O’Connor C.H., Delahunty Conor M., 2000. Food Chem. 71, 393.

van Ruth S.M., Buhr K., 2004. Int. J. Mass Spectrom. 239, 187. 